\title{
Parsonage Turner Syndrome Following COVID-19 Vaccine
}

\author{
Sohaib B. Nawaz, Wathsala A. Raigam
}

\section{ABSTRACT}

Parsonage Turner Syndrome (PTS) presents with sudden onset arm pain followed by motor weakness and can be associated with surgery, infection, or vaccination. This case discusses an incidence of PTS following COVID19 vaccination where symptoms resolved on their own progressively and extensive investigations ruled out cerebrovascular accident or vascular aetiology, and diagnosis of PTS was made. Incidence of such adverse effects is rare and awareness among clinical community can lead to recognition and prompt management of such cases.

Keywords: COVID-19 vaccination, neuralgic amyotrophy, parsonage turner syndrome, sars-cov-2 mRNA vaccine.

\section{INTRODUCTION}

Parsonage Turner syndrome also known as Neuralgic Amyotrophy or Brachial Neuritis is a compliment of symptoms where weakness in the upper limb usually proximal arm or distal forearm is preceded by sudden onset of severe unliteral shoulder and arm pain [1]. It often presents in the distribution of a nerve however can present as multiple mononeuropathies, i.e., distribution is dermatome based but involving multiple nerve roots [2]. Commonly known triggers are infection and surgery [3]. Vaccination is one of the rare triggers leading to Parsonage Turner Syndrome (PTS) [4]. Multiple trials done on safety and efficacy of COVID19 (Coronavirus Disease 2019) vaccines, specifically Severe Acute Respiratory Syndrome Coronavirus 2 (SARSCOV-2) mRNA derived Pfizer-BioNTech vaccine, did not report any significant neurological complications [5]. This case report discusses an incidence of Parsonage Turner Syndrome following immunization by SARS-COV-2 mRNA vaccine.

\section{CASE REPORT}

A 47-year-old male experienced sudden onset left sided upper limb weakness. This was coupled with severe pain in the whole of his left arm and a change of color to a bluish discoloration. The patient was carrying his grandson on his chest with both of his hands when his left arm suddenly went numb and dropped with no power. He was unable to move his arm, squeeze his hands or hold anything. The arm then started to show a bluish discoloration to it, and he then started to experience severe pain in that arm which centered over the
Submitted : Novemder 29, 2021

Published : January 19, 2022

ISSN: $2593-8339$

DOI: $10.24018 /$ ejmed.2022.4.1.1158

S. B. Nawaz*
Worcester Royal Hospital,
Worcestershire, United Kingdom.
(e-mail: Sohaib.nawaz@nhs.net)
W. A. Raigam
Worcester Royal Hospital,
Worcestershire, United Kingdom.
*Corresponding Author

left shoulder and lateral aspect of the arm (C5 nerve root). The color returned to normal after twenty minutes or so along with some movements, however, he had ongoing pain which did not start to improve till the next day. In the following $24 / 36$ hours his symptoms had greatly improved.

On presenting to his GP, palpation of his shoulder and movement of his left upper limb reproduced some of the symptoms: there was a numbness and a tingling sensation associated with a marbling color change of that limb. He was prescribed 300mg of aspirin by the GP and referred to the stroke team querying a transient ischaemic attack.

He did not experience any other neurological deficit, no problems with his speech or vision and his other limbs were normal. It was deemed unlikely to be a TIA and that there were three cardinal features of his history that pointed more towards a peripheral vascular problem: the sudden onset numbness and heaviness, the severe pain, and the discoloration of the limb. Alongside those, the reproducibility of symptoms on examination pointed more towards a vascular problem than a TIA.

On presentation to the vascular team 11 days following the onset of symptoms the patient exhibited some discomfort over his left shoulder and a degree of mild weakness of grip in his left hand. He offered no other neurological symptoms. He had a full set of palpable pulses. Further investigations were carried out to solve this diagnostic mystery.

Baseline bloods and chest x-ray were normal. A CT brain was also carried out along with bilateral carotid and left arm arterial duplex which were all unremarkable.

It was deemed that his symptoms would perhaps suggest acute brachial plexus pathology and it was queried as to whether this was a manifestation of Parsonage - Turner 
syndrome, the common association of which is a recent viral infection and less commonly immunization and interestingly this patient had received the first dose of the COVID-19

Vaccination (SARS-COV-2 mRNA derived vaccine) into his left arm two weeks prior to this event.

Further investigations in the form of a CTA Thoracic aorta and MRI spine were carried out. CTA revealed normal appearances of the thoracic aorta and its branches including the left subclavian system. MRI cervical spine showed central and left lateral disc bulge without canal stenosis at C5-6 with impingement of the exiting nerve roots. The findings on the MRI were deemed not to be relevant to the patient's presentation.

The patient was seen by the neurology team who also concluded this event to be probable neuralgic amyotrophy also known as Parsonage-Turner syndrome. The patient was followed up two and half months on from the initial event and was entirely asymptomatic with return of normal function and sensation and referred for nerve conduction studies for completion.

\section{DISCUSSION}

This case report describes an incident of Parsonage Turner Syndrome and history of presentation, progression of symptoms, and investigations carried our afterwards support the diagnosis of PTS. A 2015 study comprising 492 patients from a catchment area of population 14,118 people showed a 1-year incidence of Parsonage Turner Syndrome to be 1-in1000 [6]. The specific incidence of PTS following immunization is not well documented in the literature however it can be assumed to be rare. For example, in United States during 2018 to 2020, Vaccine Adverse Effect Reporting System had 18 incidents of Parsonage Turner Syndrome out of a large pool of 350 million who were vaccinated during that period [7]. It is yet to be seen if COVID-19 vaccinations have a higher rate of incidence of Amyotrophic Neuropathy compared to Influenza Vaccine.

The global efforts to achieve immunization to SARSCOV-2 are vital to combat COVID-10 pandemic and studies done so far provide convincing evidence of safety of these vaccines. Awareness of such rare adverse effects will help mitigate vaccine hesitancy when actual numbers documented in literature prove that such scenarios are very rare. However, there is possibility of rare occurring and more cases of Parsonage Turner Syndrome can present where timely recognition and prompt management will be helpful.

\section{DISCLAIMER}

Authors of this paper in no way support anti-vaxxer sentiment and hope that publication of this report will lead to awareness that adverse effects with COVID-19 vaccinations are rare.

\section{CONFLICT OF INTEREST}

Authors do not have any conflict of interest to express.

\section{REFERENCES}

[1] Van Eijk JJ, Groothuis JT, Van Alfen N. Neuralgic amyotrophy: an update on diagnosis, pathophysiology, and treatment. Muscle Nerve. 2016; 53(3): 337-350.

[2] Ferrante MA, Wilbourn AJ. Lesion distribution among 281 patients with sporadic neuralgic amyotrophy. Muscle Nerve. 2017; 55: 858-861.

[3] Siepmann T, Kitzler HH, Lueck C, Platzek I, Reichmann H, Barlinn $\mathrm{K}$. Neuralgic amyotrophy following infection with SARS-CoV2. Muscle Nerve. 2020; 62: E68-E70.

[4] Mahajan S, Zhang F, Mahajan A, Zimnowodzki S. Parsonage Turner syndrome after COVID-19 vaccination. Muscle Nerve. 2021; 64(1): E3-E4.

[5] Polack FP, Thomas SJ, Kitchin N, Absalon J, Gurtman A, Lockhart S, et al. Safety and efficacy of the BNT162b2 mRNA COVID-19 vaccine. New England Journal of Medicine. 2020; 383: 2603-2615.

[6] an Alfen N, van Eijk JJJ, Ennik T, Flynn SO, Nobacht IEG, Groothuis JT, et al. Incidence of Neuralgic Amyotrophy (Parsonage Turner Syndrome) in a Primary Care Setting - A Prospective Cohort Study. PLoS ONE. 2015; 10(5): e0128361.

[7] Vaccine adverse effect reporting system (VAERS). United States Department of Health and Human Services (DHHS), Public Health Service (PHS), Centers for Disease Control (CDC)/Food and Drug Administration (FDA), Vaccine Adverse Reporting System (VAERS) 1990-3/19/2021, CDC Wonder On-line Database. [Internet] 2021 $\begin{array}{llll}\text { [cited } & 2021 & \text { November } & \text { 29] }\end{array}$ from: http://wonder.cdc.gov/vaers.html.

Available

